\title{
Cognitive outcomes and age of detection of severe mucopolysaccharidosis type 1
}

\author{
Scott D. Grosse, PhD ${ }^{1}$, Wendy K.K. Lam, PhD², Lisa D. Wiggins, $\mathrm{PhD}^{1}$ and \\ Alex R. Kemper, MD, MPH²
}

The US Secretary of Health and Human Services recommended in February 2016 that mucopolysaccharidosis type 1 (MPS I) be added to the recommended uniform screening panel for state newborn screening programs. One of the key factors in this decision was the evidence suggesting that earlier treatment with hematopoietic cell transplantation (HCT) for the most severe form, Hurler syndrome (MPS IH), would lead to improved cognitive outcomes. Consistent evidence from peer-reviewed studies suggests that transplantation in the first year of life is associated with improved developmental quotient or intelligence quotient and continued cognitive growth, with earlier age of treatment associated with improved outcomes. However, available evidence suggests that cognitive functioning and attention can still lag behind unaffected age-matched children, leading to the need for special education services. Verbal and nonverbal cognitive abilities outcomes may be affected differently by HCT. With the recent addition of MPS I to the recommended uniform screening panel, future work is needed to evaluate the impact of earlier, presymptomatic detection and treatment initiation and other supportive therapies on cognitive outcomes.

Genet Med advance online publication 26 January 2017

Key Words: dried bloodspot testing; evidence-based practice; mucopolysaccharidosis type I; public health

\section{INTRODUCTION}

In February 2016, mucopolysaccharidosis type 1 (MPS I) was added to the recommended uniform screening panel (RUSP) for newborn screening by the US Secretary of Health and Human Services. This addition was based in part on a recommendation from the Advisory Committee on Heritable Disorders in Newborns and Children (hereafter, Advisory Committee), which noted that screening using dried bloodspots could effectively identify cases of the most severe phenotype in infancy and lead to long-term improved cognitive outcomes. ${ }^{1}$ Although treatment of MPS I can be lifesaving, the evidence regarding the benefit of presymptomatic detection is not supportive of a difference in mortality. However, the evidence is supportive of early treatment leading to differences in cognitive outcome. The rationale for adding MPS I to newborn screening is therefore different from that for many other metabolic disorders on the RUSP.

To evaluate MPS I for the RUSP, the Advisory Committee first considered a systematic evidence review conducted by the Condition Review Workgroup that addressed the natural history of MPS I, the accuracy of availability of screening tests, and the benefits and harms of early detection and treatment. ${ }^{1}$ That systematic evidence review was restricted to English-language articles published from 2003 through 2014, which included only one study ${ }^{2}$ that specifically addressed treatment and cognitive outcomes for the most severe form of MPS I. To further inform the Advisory Committee, the first author of our report (S.D.G.) prepared a narrative review of MPS I treatment and cognitive outcomes, which included studies published before and after the time window of the systematic evidence review. Our report provides a summary of the evidence that informed the Advisory Committee regarding the cognitive outcomes associated with MPS I and the impact of therapy on those outcomes.

\section{MPS I overview}

MPS I is an autosomal recessive lysosomal storage disorder resulting from deficiency of the enzyme $\alpha$-L-iduronidase, affecting approximately 1 in 100,000 births. ${ }^{1}$ Most children with MPS I appear normal at birth and begin to show signs or symptoms later in infancy (e.g., changes in physical appearance, inguinal or umbilical hernias, and loss of speech or learning skills). ${ }^{3}$ Traditionally, MPS I is classified as one of three phenotypes, Hurler syndrome (OMIM 607014), Hurler-Scheie syndrome (OMIM 607015), or Scheie syndrome (OMIM 60716). Hurler syndrome, also referred to as MPS IH or severe MPS $\mathrm{I}$, is the predominant form and, when untreated, is associated with rapid and progressive multisystem disease with profound central nervous system involvement in the first 2 years of life and death before 10 years of life. In contrast, Hurler-Scheie syndrome and Scheie syndrome, together referred to as attenuated MPS I (MPS IA), have later onset, slower progression,

${ }^{1}$ National Center on Birth Defects and Developmental Disabilities, Centers for Disease Control and Prevention, Atlanta, Georgia, USA; ${ }^{2}$ Duke Clinical Translational Science Institute, Duke University, Durham, North Carolina, USA. Correspondence: Alex R. Kemper (alex.kemper@duke.edu) 
and typically little or no central nervous system involvement. Untreated, Hurler-Scheie syndrome is associated with death in the second or third decade of life. In contrast, most individuals with Scheie syndrome have a normal lifespan. Enzyme replacement therapy (ERT) with laronidase is available for MPS I. However, because laronidase does not penetrate the bloodbrain barrier, hematopoietic cell transplantation (HCT) is recommended for MPS IH in addition to ERT. ${ }^{4}$ Guidelines recommend HCT for patients with MPS IH who are younger than 30 months old and have an estimated developmental quotient (DQ) of at least 70. These restrictions are based on the expected benefits of HCT on long-term outcomes and overall posttransplant event-free survival, which is estimated to be $90 \%{ }^{5}$

\section{Natural history of cognitive outcomes in MPS I}

Untreated children with MPS IH develop progressive cognitive decline relative to age-specific norms beginning at approximately 6 months of age, ${ }^{6}$ and the degree of cognitive impairment increases over time. Among a series of 15 untreated children with MPS IH, the median DQ decreased from an average of 80 (below average) prior to 2 years of age to 61 (well below average) after 2 years of age. ${ }^{7}$ After excluding two outliers, the correlation between DQ and age in years was -0.82 . A more recent report of 22 untreated children, including the 15 previously described, found a mean IQ of 67 (well below average) at a mean age of 2.28 years. ${ }^{8}$ Across the population, the IQ decreased with increasing age, and all died in early childhood.

In general, natural history studies have not reported cognitive impairment among untreated children with MPS IA. However, treatment studies suggest that cognitive impairment might be an important feature of the condition.

\section{Treatment and cognitive outcomes for MPS IH}

Natural history studies show that untreated MPS I is associated with cognitive declines that result in cognitive skills that are well below average (i.e., mean DQ scores between 61 and 67 points in two studies). ${ }^{7.8}$ Past research has shown that HCT prevents progressive deterioration in cognitive outcomes into the extremely low average range among children with MPS $\mathrm{IH},{ }^{9,10}$ although cognitive functioning remains below average for some children compared with same-aged peers. Regardless of statistical significance or comparisons with other groups, clinically meaningful cognitive improvement may be demonstrated with HCT treatment. Moderate effect sizes $(\geq 0.50$ standard deviation) or approximately seven standardized test points after HCT treatment (e.g., DQ scores between 68 and 74 points when compared to previous studies) are likely to yield meaningful benefit for the patient and/or family. ${ }^{11}$ Three studies published in the 1990s reported that after HCT, only approximately half had no deterioration or improved cognitive performance; the remaining had lower cognitive scores. ${ }^{12-14}$ More recently, Eisengart and colleagues ${ }^{15}$ reported that among 10 patients who received HCT but no ERT, at a mean age of 17 months the mean overall cognitive composite standard score was 91 (average) before transplantation and declined during the 2-year follow-up period to a mean of 71 points (below average). Children who received HCT had a significantly higher rate of decline in the visual reception domain-a subscale measure of nonverbal learning abilities - than children who received both HCT and ERT.

Aldenhoven and colleagues ${ }^{16}$ reported data for 217 patients with MPS IH who underwent successful engrafting following HCT at 10 centers worldwide between 1985 and 2011. Overall, patients underwent transplantation at a median age of 16 months, with last follow-up at a median of 110 months. The prevalence of below-average cognitive functioning (DQ or IQ $<85)$ in this sample increased from $43 \%$ prior to HCT to $73 \%$ at last follow-up. The prevalence of intellectual disability (DQ or IQ <70) increased from 17 to $45 \%$. Male sex, lower baseline DQ or IQ, older age at HCT, use of total body irradiation, and older age at evaluation were each associated with poorer cognitive outcomes after HCT.

Shapiro and colleagues ${ }^{8}$ reported cross-sectional assessments for 60 patients with MPS IH treated at five US and Canadian centers. Fourteen children assessed pre-HCT ( $\leq$ age 24 months), had a mean age of treatment of 7.5 months, and a mean pre-HCT IQ score of 91 (average range). Among 46 children and young adults assessed between $2-25$ years of age, mean age of treatment was 11.4 to 12.6 months, with IQ scores averaging 76 (below average range). Of the 14 children in the pre-HCT sample, $7 \%$ had intellectual disability (IQ $<70) ; 37-42 \%$ of the 46 children in the older age groups who had undergone transplantation years earlier had intellectual disability. Among children treated with HCT, there was a difference of 9 points between the children's verbal IQ scores before and after HCT and a difference of 14 points between the children's nonverbal IQ scores before and after HCT. The strongest predictors of cognitive outcome were genotype and age at HCT. More than $90 \%$ of children required special education or therapy services after HCT. The authors concluded that HCT is associated with stabilization of cognitive ability rather than continued progressive decline in MPS IH patients, even though below-average functioning remains prominent.

Two smaller studies reported more positive post-HCT cognitive outcomes. Souillet ${ }^{17}$ followed 15 children with MPS IH who underwent transplantation at ages 14-51 months for at least 36 months after HCT. All children experienced progress after HCT but later showed decreases in age-equivalent cognitive scores, followed by apparent stabilization. None of the subjects had intellectual disability (IQ <70). However, most had reported problems with concentration, memory, and language, and only one in five children older than age 12 years did not require special services at school. Another study ${ }^{18}$ similarly reported motor and language delays in six children with MPS IH who underwent transplantation before 24 months, followed by continued cognitive and/or language delays in four of six children 24 months after transplantation, and then stabilization of cognitive development before reaching school age. 


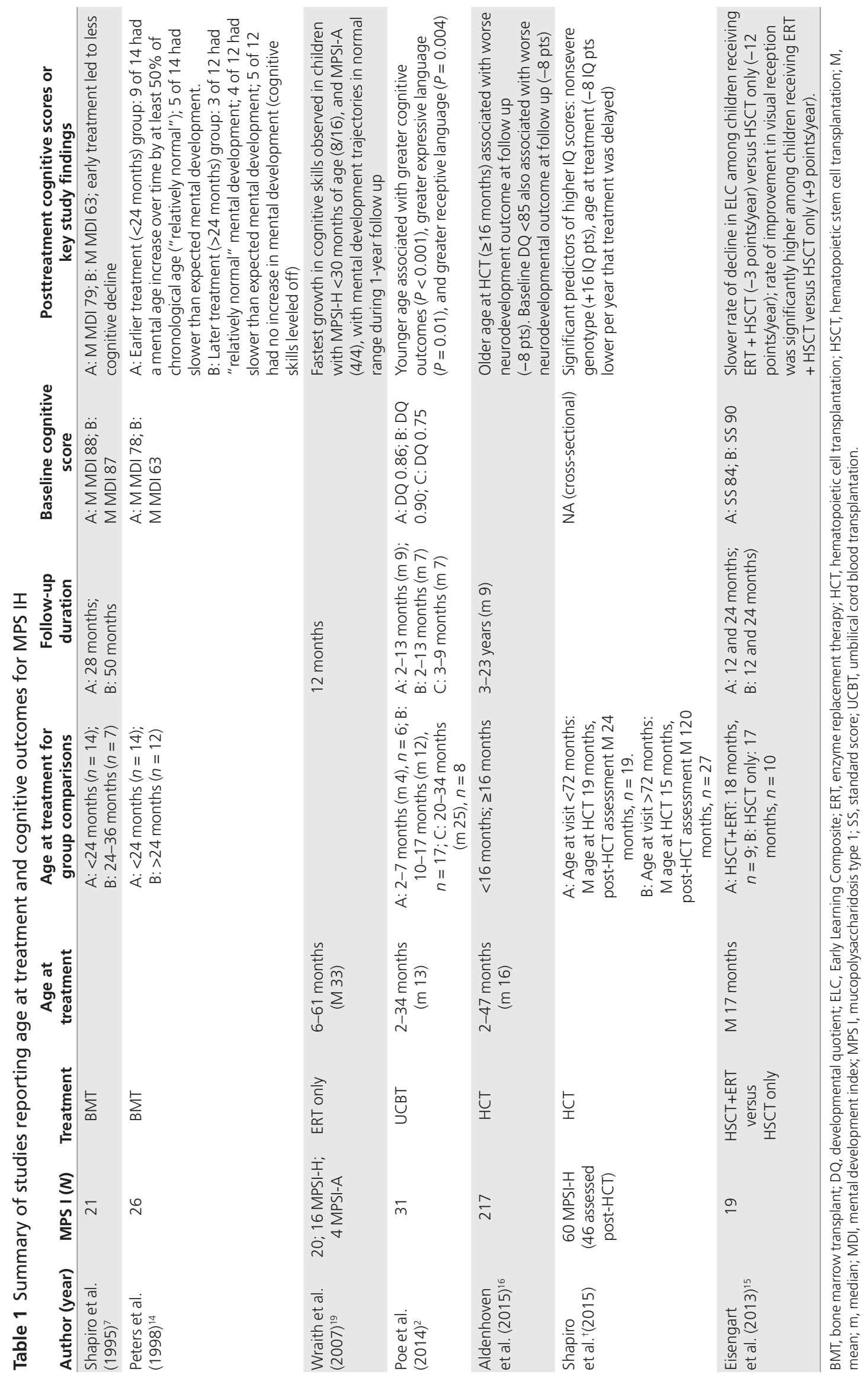




\section{Age of treatment and cognitive outcomes for MPS IH}

One of the most consistent findings in the recent MPS I literature is that younger age at HCT treatment is associated with improved cognitive outcomes. These studies are reviewed here and summarized in Table 1. A 1995 report by Shapiro and colleagues ${ }^{7}$ analyzed data for 21 children with MPS IH without baseline intellectual disability (DQ $>70$ ) and followed at least 12 months after transplantation. The mean decrease in standard scores was 4 points per year for children who underwent transplantation before 24 months and 7 points per year for children who underwent transplantation between 24 and 36 months of age. Mean scores decreased by 9 points, from 88 (average) to 79 (below average), for the early-transplant group (mean, 28 months of follow-up) and by 24 points, from 87 (average) to 63 (well below average), for the late-transplant group (mean, 50 months of follow-up). The percentages with intellectual disability (standard score <70) were 29\% (four of 14) for the group who underwent transplantation before age 24 months and 57\% (four of seven children) for the group transplanted between ages 24 and 36 months. The percentages with severe intellectual disability (standard score $<50$ ) were $7 \%$ (one of 14 ) for the group who underwent transplantation before age 24 months and $57 \%$ (four of seven) for the group transplanted between ages 24 and 36 months.

Peters et al. ${ }^{14}$ also reported cognitive outcomes stratified by age at transplantation for those younger than 24 months and those older than 24 months. Of 14 children who underwent transplantation when they were younger than 24 months, 9 were reported to have had "relatively normal" development, meaning that mental age increased over time by at least $50 \%$ of the chronological age, and 5 experienced slower than expected development. Of 12 children who were transplanted after age 24 months, three had "relatively normal" development, four had slower than expected development, and five children plateaued in development or failed to progress further. The mean difference in cognitive growth trajectories between children who underwent transplantation before and after age 24 months was significant and indicated that children transplanted before age 24 months showed more cognitive progression than children transplanted after age 24 months. Moreover, children who underwent transplantation before age 24 months were less likely to plateau or show a leveling off of cognitive skills than children transplanted after 24 months.

Wraith and colleagues ${ }^{19}$ reported cognitive growth outcomes for 20 children between 6 and 61 months of age with MPS I who were enrolled in a year-long open-label trial of ERT without HCT. The fastest growth in cognitive skills occurred among the eight children with MPS IH who were younger than 30 months and all four of the children with MPS IA regardless of age. Children with MPS IH who were older than 30 months showed a developmental plateau in cognitive skills. The authors noted that longer follow-up is needed to determine whether developmental gains made by children with MPS IH who were younger than 30 months at the time of enrollment are sustained.
Poe and colleagues ${ }^{2}$ enrolled 31 patients who underwent transplantation at two US institutions between 1997 and 2013. DQ scores were calculated before HCT and at least two times after HCT, with a median of seven subsequent evaluations. A longitudinal regression analysis was used to evaluate the relationship between age at transplantation and cognitive outcome. Younger age at transplantation was associated with better long-term cognitive outcomes $(P<0.001)$, with each month of transplantation delay associated with a greater deficit in longterm outcomes. Moreover, children who underwent transplantation at younger ages had significantly better subscale scores for expressive and receptive language functions than those transplanted at later ages ( $P=0.01$ and 0.004 , respectively). The authors did not report nonverbal cognitive skills independent of language function.

The study investigators also reported individual and modelgenerated average trajectories stratified by age at transplantation. ${ }^{2}$ Results showed that individual children in the late-transplant group were more likely to perform at the lowest developmental levels and were more likely to plateau in development, although there was no evidence of further deterioration after a posttransplantation decline. In contrast, the two groups who underwent transplantation before 18 months of age showed development within the normal range. The earliest transplant group (ages 2-8 months) showed normal cognitive, expressive language, and receptive language development, with the average trajectory at or near the 50th percentile in normal populations. In a fixed-effects model, the children who underwent transplantation at 2-8 months had significantly better cognitive function than those transplanted after 8 months $(P=$ 0.001 ). The group transplanted between ages 9 and 17 months performed, on average, below the 50th percentile but within 2 standard deviations of the normal population distribution in all developmental domains. The group who underwent transplantation after 18 months performed, on average, more than 2 standard deviations below the mean in all developmental domains.

Aldenhoven and colleagues ${ }^{16}$ charted average developmental trajectories for 217 children who underwent transplantation at 10 centers in Europe and the United States from 1985 through 2011 and were stratified separately by pre-HCT DQ and age at HCT. Those transplanted before the median age of 16 months had higher developmental ages throughout follow-up relative to those transplanted after 16 months. The regression analysis indicated that later transplantation was associated with an 8.4point reduction in cognitive scores at last follow-up. Baseline DQ $<85$ was associated with an 8.6-point reduction in cognitive scores at last follow-up. The authors concluded that early diagnosis through newborn screening is likely to result in better cognitive outcomes for children with MPS IH in two ways. First, younger age of HCT directly predicts better cognitive test scores. Second, younger age at HCT is associated with higher baseline DQ at the time of HCT, which is also an important predictor of neurodevelopment in children with MPS IH. 


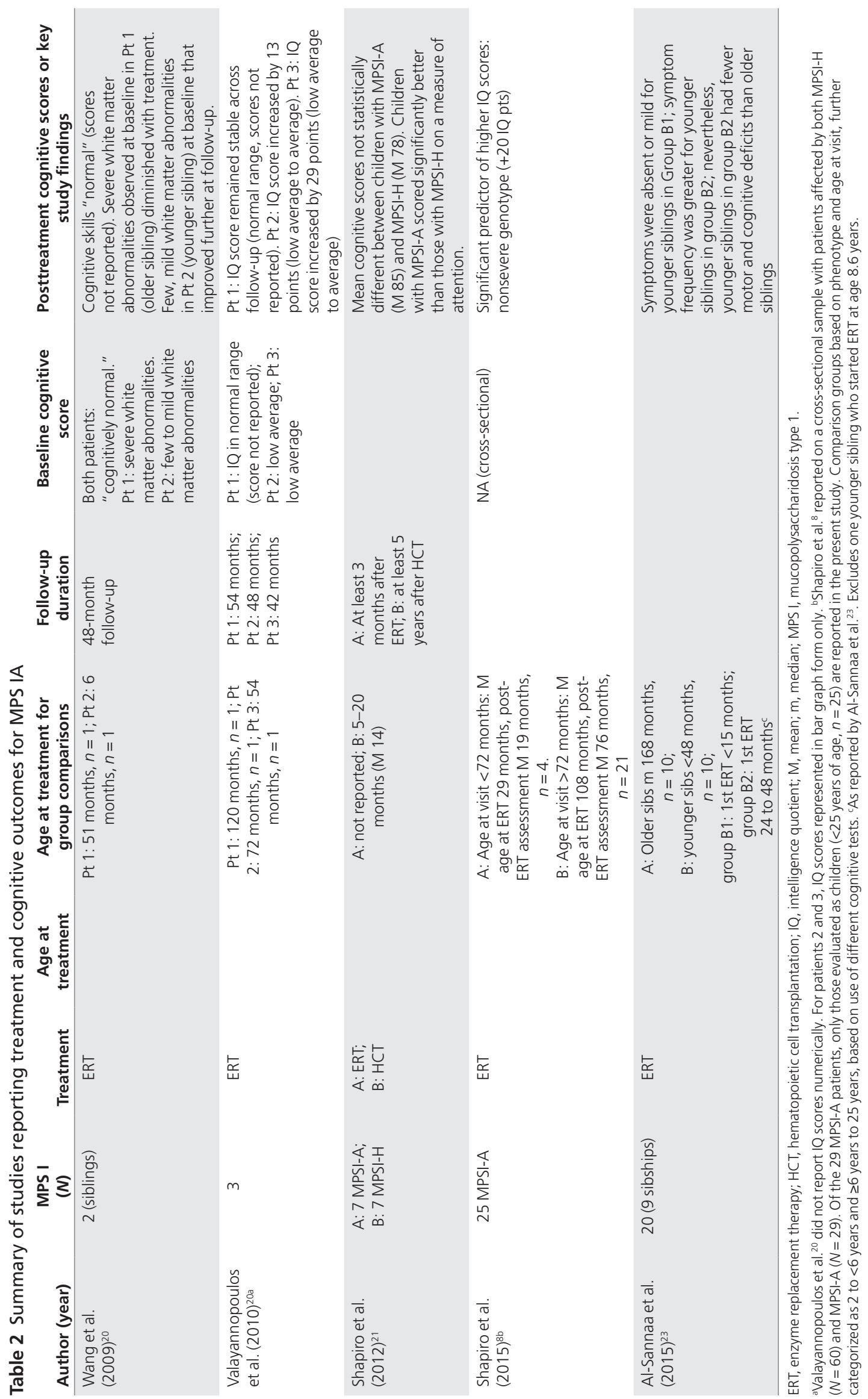


Shapiro and colleagues ${ }^{8}$ analyzed cross-sectional data for 60 patients with MPS IH, of whom 14 were administered ERT prior to HCT and 46 were assessed post-HCT. Of those assessed post-HCT, 19 were assessed at ages $24-71$ months and 27 after 71 months. A regression analysis found that the most powerful predictor of IQ scores was genotype, with nonsevere mutations associated with scores higher by approximately 20 points. Age at treatment was also significant, with an average 8-point decrease in cognitive scores for each year treatment was delayed. The authors also noted that patients who underwent a second HCT had lower IQ scores.

\section{Treatment and cognitive outcomes for MPS IA}

A small number of studies have reported cognitive outcomes for children with MPS IA who were treated by ERT. Until very recently, no study had tested the hypothesis that earlier diagnosis and treatment of MPS IA resulted in fewer brain abnormalities and improved cognitive outcomes. These studies of cognitive outcomes among children with MPS IA who were treated with ERT are reviewed here and are summarized in Table 2.

Wang and colleagues ${ }^{20}$ reported outcomes for two siblings who were diagnosed at approximately the same time when they were 48 and 3 months of age, and who each started ERT 3 months after diagnosis. Although both children were in the normal cognitive range (no test scores reported), magnetic resonance imaging (MRI) tests conducted before and after 48 months of treatment showed extensive white matter abnormalities in the older sibling that diminished with treatment, but the young sibling had few abnormalities at either point in time.

Valayannopoulos and colleagues ${ }^{21}$ reported both cognitive and MRI outcomes for three unrelated children (Patients 1, 2, and 3) who had late initiation of ERT following diagnosis at ages 42,72 , and 120 months of age. All three had full-scale IQ test scores in the normal range at baseline, but the two children diagnosed at younger ages had significant improvements over 30-36 months of treatment, with IQ gains of 13 and 29 points. Gains in the verbal and nonverbal domains were evident. Patient 2 had significant academic problems that resolved following treatment. MRI scans of patients 2 and 3 showed significant improvements in white matter involvement, whereas patient 1 had no significant abnormalities at baseline.

Another study by Shapiro and colleagues ${ }^{22}$ reported cognitive outcomes for seven children with MPS IA tested at a mean age of 16 years who had been administered ERT for a maximum of 6 years. The mean cognitive score was 85 (low average). The authors concluded that the below-average mean IQ in the MPS IA group "dispels any notion that their CNS is entirely intact." Moreover, the authors suggested that the cognitive limitations seen in MPS IA patients may warrant educational and vocational modifications.

A more recent study ${ }^{8}$ reported more extensive cross-sectional data for 21 patients with MPS IA who were 72 months or older (mean age, 15.5 years) as well as 4 patients with MPS IA younger than 72 months (mean age, 4.3 years) at the time of evaluation.
Mean full-scale IQ scores were 91 and 109, respectively, and the percentages with full-scale IQ in the average range were $57 \%$ and $100 \%$, respectively. There was an 18-point difference in verbal IQ between MPS IA children who were assessed at younger ages ( $\leq 72$ months) compared with those assessed at older ages (>72 months), with the younger children performing more than 1 standard deviation better than those assessed at later ages. Similarly, there was a 13-point difference in nonverbal IQ between these two groups, with the younger children with MPS IA performing better than those with MPS IA detected later. The predominant predictor of IQ score was genotype, and having one severe mutation was associated with a 20-point lower IQ score. Almost $30 \%$ of the patients 72 months of age and older had a severe mutation compared with none of the younger patients. In a regression equation that controlled for genotype and somatic symptoms, age at initiation of treatment had a modest effect that was not significant at the 0.05 level, with each 12-month delay in initiation of treatment associated with a 2-point lower IQ score.

The influence of genotype on MPS IA outcomes has been reported elsewhere. One study ${ }^{23}$ identified a subset of individuals with MPS IA who were at risk for both psychiatric problems and cognitive impairment, specifically those carrying an L238Q missense mutation. In a sample of 14 patients with MPS IA who were ages $15-25$ years, the mean IQ score for six L238Q carriers was 74 (below average) compared with 95 (average) for non-L238Q carriers $(P=0.016)$. These results support those of Shapiro and colleagues and suggest that genotype may be an important consideration when considering developmental trajectory among patients with MPS I.

Al-Sannaa and colleagues ${ }^{24}$ used a sample of 20 patients with MPS IA from nine affected sibships in seven countries to assess the impact of treatment on younger siblings before disease manifestation. Younger siblings were stratified by age at ERT initiation to gain further insight into the influence of early diagnosis and treatment. Symptoms were absent or mild for younger siblings who started ERT at age 12 months or younger. Younger siblings who started ERT between ages 24 and 48 months had more motor and cognitive deficits and more restriction in activities of daily living than the younger siblings who started ERT earlier in life ( $\leq 12$ months). Younger siblings who started ERT at age younger than 12 months had fewer motor and cognitive deficits than their older siblings. In summary, these results suggest that ERT treatment before disease manifestation can attenuate cognitive declines, especially among children who initiate treatment in the first few years of life.

\section{DISCUSSION}

The Advisory Committee recommended that MPS I be added to the RUSP because of the potential cognitive benefit of early identification through newborn screening. Cognitive scores decrease with increasing age among untreated children with MPS IH, and this review supports the hypothesis that earlier age of treatment is associated with significantly improved cognitive function. We found studies supporting that HCT at 
younger than 9 or 16 months of age was associated with significantly better cognitive outcomes and lower risk of cognitive impairment, ${ }^{2,16}$ and that a 12-month delay in HCT can reduce final IQ scores by approximately 8 points after controlling for genotype. ${ }^{8}$ Overall, early HCT treatment prevents further cognitive decline and allows for stabilization and potential growth in cognitive skills. ${ }^{2,14,19}$

Poe et al., ${ }^{2}$ along with others using data from the same patient cohorts, ${ }^{25,26}$ found substantially more favorable long-term cognitive outcomes following early HCT than either the longitudinal study by Aldenhoven and colleagues ${ }^{16}$ or the cross-sectional study by Shapiro and colleagues. ${ }^{8}$ The differences in outcomes might be attributable to younger age at HCT (2-8 months) ${ }^{2}$ versus $2-47$ months ${ }^{16}$ or $24-71$ months. ${ }^{8}$ In addition, the differences might reflect improvements in treatment over time (HCT between 1997 and $2013^{2}$ versus between 1995 and $2006^{26}$ and between 1985 and 2011). ${ }^{16}$ Reports of transplantations performed most recently ${ }^{2}$ used standardized transplant protocols and methods of developmental assessment; therefore, they were less heterogeneous than previous studies. ERT, whether alone or in combination with HCT, appears to attenuate cognitive decline in MPS I. The mechanism of attenuation is unclear because ERT does not cross the blood-brain barrier. ${ }^{27,28}$ One prospective study indicates that among children with MPS IH who were treated for 12 months with ERT alone, treatment before 18 months was associated with less deterioration in cognitive function..$^{19}$ Another prospective study compared children with MPS IH who received either HCT alone or ERT with HCT; the decline in DQ scores during 24 months of follow-up was much greater for the HCT only group. ${ }^{15}$

Mild cognitive impairment is common among children with MPS IA, ${ }^{8,22}$ particularly in association with the L238Q missense mutation. ${ }^{8,23}$ Cognitive outcomes for MPS IA merit further attention by researchers. In particular, researchers might investigate the impact of verbal versus nonverbal abilities and attention skills on cognitive measures and whether earlier initiation of ERT for children with MPS IA associated with the L238Q missense mutation leads to less cognitive impairment.

Although language development appears to benefit from earlier treatment ${ }^{2}$ and can normalize over time ${ }^{18}$ cognitive and attention skills can remain suboptimal for children regardless of the age at HCT. ${ }^{17}$ Many children with MPS IH require special education services even after HCT. ${ }^{8,17}$ Even after treatment, children with MPS IH typically perform worse than those with MPS IA on measures of spatial judgment and attention. ${ }^{22}$

Intellectual function and academic performance depend on the complex relationship between verbal, nonverbal, and attention skills. Although early treatment for MPS IH leads to improved cognitive outcomes overall, treatment can still leave important gaps in overall function. Future work is needed to identify other supportive therapies that could target specific academic and cognitive domains that are not directly improved with early treatment with HCT or ERT.

This review identified significant research gaps related to developmental outcomes in MPS I. Additional analyses of existing data that have already been published could yield valuable information. For example, it would be useful to test whether there might be a nonlinear association of IQ with age at treatment rather than assume a linear regression. Similarly, investigators that used a single age cutoff for initiation of treatment could revisit their data to determine how use of a different age cutoff might alter results. Finally, it might be helpful to stratify data by time period to investigate whether improvements in clinical practice over time might have altered the association of age of treatment with cognitive outcomes.

The rarity of MPS I makes it difficult to assess and understand the relationship between age at specific treatments and developmental outcomes. The MPS I Disease Registry (https:// www.mps1disease.com/en/patients/mpsi-registry.aspx) could play an important role in improving outcomes through better knowledge regarding expected outcomes and the relative benefit of different treatment strategies if standardized measures of intellectual function are collected at common ages. A registry approach may be especially beneficial to understanding the developmental trajectory of and treatment response among the small number of identified MPS IA patients. Important considerations for both MPS IH and MPS IA include long-term patterns of change over time in verbal, nonverbal, and attention scores based on patient diagnosis and specific treatment and the role of other supportive interventions.

\section{Conclusion}

In summary, consistent evidence from multiple peer-reviewed studies indicates that earlier initiation of treatment (ERT and/ or HCT) for children with MPS IH is associated with markedly better cognitive functioning overall. Transplantation in the first year, preferably as early as possible, is a particularly strong predictor of improved cognitive performance and continued cognitive growth. ${ }^{2,24}$ This review did not assess impacts on subscale results by developmental domain. Future analyses should consider assessments of differential impacts of MPS I treatment on verbal versus nonverbal cognitive development, academic skills, and attention skills. Also, more work is needed to understand the different cognitive trajectories for patients with MPS IA, an understudied patient group.

\section{ACKNOWLEDGMENT}

The authors acknowledge helpful comments from Maria L. Escolar and Michelle D. Poe regarding the interpretation of their studies. This project was supported by the Health Resources and Services Administration (HRSA) of the US Department of Health and Human Services (HHS) under contract HHSH250201400007C, titled "Evidence-based Reviews for the Discretionary Advisory Committee on Heritable Disorders in Newborns and Children."

The views expressed herein are solely those of the individual authors and do not necessarily reflect the views of the Secretary of the United States Department of Health and Human Services or the Centers for Disease Control and Prevention. This information or content and conclusions are those of the authors and should not be construed as the official position or policy of, nor should any endorsements be inferred by, HRSA, HHS, or the US Government. 


\section{DISCLOSURE}

The authors declare no conflict of interest.

\section{REFERENCES}

1. Kemper AR, Brosco, J, Comeau, AM, et al. Newborn Screening for Mucopolysaccharidosis Type 1 (MPS I): A Systematic Review of Evidence Report of Final Findings. 16 March 2015. http://www.hrsa.gov/advisorycommittees/ mchbadvisory/heritabledisorders/nominatecondition/reviews/mps1 finalreport. pdf. Accessed 26 July 2016.

2. Poe MD, Chagnon SL, Escolar ML. Early treatment is associated with improved cognition in Hurler syndrome. Ann Neurol 2014;76:747-753.

3. Muenzer J. The mucopolysaccharidoses: a heterogeneous group of disorders with variable pediatric presentations. J Pediatr 2004;144(5 suppl):S27-S34.

4. Muenzer J, Wraith JE, Clarke LA; International Consensus Panel on Management and Treatment of Mucopolysaccharidosis I. Mucopolysaccharidosis I: management and treatment guidelines. Pediatrics 2009;123:19-29.

5. Aldenhoven M, Jones SA, Bonney D, et al. Hematopoietic cell transplantation for mucopolysaccharidosis patients is safe and effective: results after implementation of international guidelines. Biol Blood Marrow Transplant 2015;21:1106-1109.

6. Aldenhoven M, Boelens JJ, de Koning TJ. The clinical outcome of Hurler syndrome after stem cell transplantation. Biol Blood Marrow Transplant 2008;14:485-498.

7. Shapiro EG, Lockman LA, Balthazor M, Krivit W. Neuropsychological outcomes of several storage diseases with and without bone marrow transplantation. J Inherit Metab Dis 1995;18:413-429.

8. Shapiro EG, Nestrasil I, Rudser K, et al. Neurocognition across the spectrum of mucopolysaccharidosis type I: Age, severity, and treatment. Mol Genet Metab 2015;116:61-68.

9. Boelens JJ, Prasad VK, Tolar J, Wynn RF, Peters C. Current international perspectives on hematopoietic stem cell transplantation for inherited metabolic disorders. Pediatr Clin North Am 2010;57:123-145.

10. Boelens JJ, Orchard PJ, Wynn RF. Transplantation in inborn errors of metabolism: current considerations and future perspectives. Br J Haematol 2014;167: 293-303.

11. Cohen J. Statistical Power Analysis for the Behavioral Sciences, Rev. Hillsdale, NJ: Lawrence Erlbaum Associates; 1977.

12. Vellodi A, Young EP, Cooper A, et al. Bone marrow transplantation for mucopolysaccharidosis type I: experience of two British centres. Arch Dis Child 1997;76:92-99.

13. Peters C, Balthazor M, Shapiro EG, et al. Outcome of unrelated donor bone marrow transplantation in 40 children with Hurler syndrome. Blood 1996;87:4894-4902.
14. Peters C, Shapiro EG, Anderson J, et al. The Storage Disease Collaborative Study Group. Hurler syndrome: II. Outcome of HLA-genotypically identical sibling and HLA-haploidentical related donor bone marrow transplantation in fifty-four children. Blood 1998;91:2601-2608.

15. Eisengart JB, Rudser KD, Tolar J, et al. Enzyme replacement is associated with better cognitive outcomes after transplant in Hurler syndrome. J Pediatr 2013;162:375-80.e1.

16. Aldenhoven M, Wynn RF, Orchard PJ, et al. Long-term outcome of Hurler syndrome patients after hematopoietic cell transplantation: an international multicenter study. Blood 2015;125:2164-2172.

17. Souillet G, Guffon N, Maire I, et al. Outcome of 27 patients with Hurler's syndrome transplanted from either related or unrelated haematopoietic stem cell sources. Bone Marrow Transplant 2003;31:1105-1117.

18. Malm G, Gustafsson B, Berglund G, et al. Outcome in six children with mucopolysaccharidosis type $\mathrm{IH}$, Hurler syndrome, after haematopoietic stem cell transplantation (HSCT). Acta Paediatr 2008;97:1108-1112.

19. Wraith JE, Beck M, Lane R, et al. Enzyme replacement therapy in patients who have mucopolysaccharidosis I and are younger than 5 years: results of a multinational study of recombinant human alpha---iduronidase (laronidase). Pediatrics 2007;120:e37-e46.

20. Wang RY, Cambray-Forker EJ, Ohanian K, et al. Treatment reduces or stabilizes brain imaging abnormalities in patients with MPS I and II. Mol Genet Metab 2009;98:406-411.

21. Valayannopoulos V, Boddaert N, Barbier V, Le Merrer M, Caillaud C, de Lonlay P. Cognitive and neuroradiological improvement in three patients with attenuated MPS I treated by laronidase. Mol Genet Metab 2010;100:20-23.

22. Shapiro E, Guler OE, Rudser K, et al. An exploratory study of brain function and structure in mucopolysaccharidosis type l: long term observations following hematopoietic cell transplantation (HCT). Mol Genet Metab 2012;107: 116-121.

23. Ahmed A, Whitley CB, Cooksley R, et al. Neurocognitive and neuropsychiatric phenotypes associated with the mutation L238Q of the $\alpha$-L-iduronidase gene in Hurler-Scheie syndrome. Mol Genet Metab 2014;111:123-127.

24. Al-Sannaa NA, Bay L, Barbouth DS, et al. Early treatment with laronidase improves clinical outcomes in patients with attenuated MPS I: a retrospective case series analysis of nine sibships. Orphanet J Rare Dis 2015;10:131.

25. Staba SL, Escolar ML, Poe M, et al. Cord-blood transplants from unrelated donors in patients with Hurler's syndrome. N Eng/ J Med 2004;350:1960-1969.

26. Coletti HY, Aldenhoven M, Yelin K, Poe MD, Kurtzberg J, Escolar ML. Long-term functional outcomes of children with hurler syndrome treated with unrelated umbilical cord blood transplantation. JIMD Rep 2015;20:77-86.

27. Pastores GM. Laronidase (Aldurazyme): enzyme replacement therapy for mucopolysaccharidosis type I. Expert Opin Biol Ther 2008;8:1003-1009.

28. Muenzer J. Early initiation of enzyme replacement therapy for the mucopolysaccharidoses. Mol Genet Metab 2014;111:63-72. 